\title{
Valor pronóstico de la ecocardiografía de ejercicio en la enfermedad coronaria
}

Use of exercise echocardiography for prognostic evaluation of patients with known or suspected coronary artery disease.

Marwick T, Mehta R, Arheart C et al. J Am Coll Cardiol 1997;30:83-90.

\section{Objetivo}

Determinar el valor pronóstico adicional de la ecocardiografía de ejercicio (EE) con respecto a la prueba ergométrica graduada (PEG).

\section{Diseño}

Estudio prospectivo, de cohorte. El seguimiento fue de $44+/$ - 11 meses.

\author{
Lugar \\ Cleveland Clinic, EE.UU.
}

\section{Participantes}

Se incluyeron 500 pacientes consecutivos, edad $57+/-12$ años. El $53 \%$ tenía diagnóstico de angor crónico estable y el $47 \%$, sospecha de enfermedad coronaria.

\section{Evaluación de factores pronósticos}

Se analizó el valor predictor independiente de distintas variables: clínicas (antecedente de infarto agudo de miocardio -IAM- y factores de riesgo); ergométricas (capacidad funcional, doble producto, frecuencia cardíaca -FC-, \% de la FC máxima prevista, infradesnivel del ST, angor) y ecocardiográficas (isquemia, infarto ó ambos).

\section{Medición de resultados principales}

a) Eventos totales (punto final combinado): muerte, IAM, angina inestable ó revascularización efectuada más de 3 meses después de realizado el estudio. (Para evitar el sesgo de indicación terapéutica basada en los resultados del estudio).

b) Muerte, IAM ó angina inestable combinados

\section{Resultados Principales}

El 93\% de los pacientes completaron el seguimiento $(44+/-11$ meses). La PEG fue positiva en 160 pacientes (32\%) y no interpretable en 54 (11\%). La EE fue positiva en 200 pacientes (40\%). a) Eventos totales: $n=81(17 \%)$

Sólo demostraron valor predictor independiente ( $\mathrm{p} 0.001)$ : porcentaje de FC máxima alcanzada según edad (RR por cada $5 \%$ de aumento 0.84 , IC 95\% 0.77-0.92) y evidencia de isquemia en el EE (RR 5.06, IC $95 \% 3.09-8.29$ )

b) Muerte, IAM ó angina inestable combinados: $n=33(7 \%)$ Mostró valor predictor independiente la presencia de isquemia en el EE (p.0001, RR 8.2, IC 95\% 4.4 - 19.7) y el porcentaje de FC máxima alcanzada según edad (RR por cada $5 \%$ de aumento 0.78 , IC $95 \%$ $0.67-0.91)$.

\section{Conclusiones}

La detección de isquemia en el ecocardiograma de ejercicio tiene valor pronóstico independiente y aditivo con respecto a la información suministrada por la prueba ergométrica.

\section{COMENTARIO}

Se han publicado diversos artículos que demuestran el valor pronóstico del ecocardiograma de ejercicio en la enfermedad coronaria (1). Sin embargo, queda por determinar cuál es la población de pacientes en la que se justificaría la utilización de un método más costoso que la prueba ergométrica.

En este estudio, la detección de isquemia por ecocardiografía se asoció a una mayor tasa de eventos. Sin embargo, la interpretación de los resultados obtenidos resulta confusa debido a los siguientes hechos: 1) En cuanto a la selección de la población, sólo la mitad de los pacientes tenían una enfermedad coronaria conocida, mientras que el resto había sido estudiado con finalidad diagnóstica. El pronóstico de estos dos grupos es diferente y resulta esperable que la utilidad del test para evaluarlo también lo sea, a pesar de lo cuál han sido analizados en forma conjunta.

2) En la medición de los resultados se utilizó un punto final combinado que incluye eventos de muy distinta relevancia clínica, tales como muerte, infarto, angor inestable o necesidad de revascularización.

3) La tasa de eventos a 5 años fue relativamente baja, lo cuál podría deberse a la inclusión de dos poblaciones con diferente riesgo, a la exclusión de los pacientes revascularizados precozmente y a las características del $7 \%$ de los pacientes perdidos en el seguimiento (la mitad tenían antecedente de IAM y las tres cuartas partes presentaban isquemia en el ecocardiograma).

4) El resultado negativo del ecocardiograma predijo una tasa de eventos muy baja ( $5 \%$ a los 2 años) y, si bien no se mencionan los valores correspondientes a la prueba ergométrica aislada, es conocido el valor de la misma en la identificación de pacientes de bajo riesgo, con cifras similares a las anteriores.

5) Hay un $11 \%$ de PEG ininterpretables: sería interesante conocer el valor predictor del ecocardiograma en estos pacientes (lo cuál no fue analizado), ya que probablemente el método sea de mayor utilidad en este subgrupo.

Dado que este estudio no cambia la conducta hasta el momento, la EE se reserva para los pacientes cuyo electrocardiograma sea de difícil interpretación (trastornos de conducción, drogas, sobrecarga ventricular, etc.); cuando se requiera evaluar la viabilidad miocárdica; y cuando la conducta terapéutica dependa de la localización y extensión de la isquemia (identificación del vaso responsable).

\section{Dra. Vilma E. Irazola.}

Servicio de Cardiología. Hospital de Clínicas José de San Martín. UBA. 